\title{
Scattering of partially coherent surface plasmon polariton fields by metallic nanostripe
}

\author{
Salman Daniel ${ }^{1 *}$, Kimmo Saastamoinen ${ }^{1}$, Sergey A. Ponomarenko ${ }^{2,3}$ and Ari T. Friberg ${ }^{1}$
}

\begin{abstract}
Background: Surface plasmon polaritons (SPPS) are electromagnetic waves that propagate at a metal-dielectric interface. Until recently, monochromatic, fully coherent SPPs have mainly been considered.

Methods: We investigate by numerical simulations the generation and properties of polychromatic, partially coherent SPPs and their scattering from a nanostripe. We use both in-house and commercial codes.

Results: A standing SPP field is created in Kretschmann's setup between the excitation point and the nanostripe. From the scattered far-field spectrum, all spatiotemporal coherence properties of the SPP field pattern can be deduced. Illustrative examples of such coherence variations are analyzed.
\end{abstract}

Conclusions: Plasmonic nanostructures produce strong confined SPP fields of widely controllable coherence.

Keywords: Surface plasmon polaritons, Partial coherence, Nanoscattering

\section{Background}

Surface plasmon polaritons (SPPs) are evanescent electromagnetic waves that propagate along an interface between a metal and a dielectric medium $[1,2]$. Characterized by strong lateral confinement and large propagation lengths, SPPs have numerous applications in near-field optics and nanophotonics, including plasmonic waveguides and switches, biosensors, and data storage devices [3-5]. They allow to scale down optical systems to nanometer dimensions [6]. SPPs can be generated at a metal-dielectric interface by several techniques, among them the Kretschmann and Otto configurations [1,2], metallic grating coupling [7], and confined fields [2]. Fulfilment of a wave vector phase-matching condition is required in most setups. On interaction with subwavelength features, such as grooves or nanoslits, the SPPs convert back to freely propagating optical waves [8]. Over the years, a vast amount of research has been conducted to investigate the fundamental problem of SPP scattering by small structures and defects (see, e.g., [9-11]).

*Correspondence: salman.daniel@uef.fi

${ }^{1}$ Institute of Photonics, University of Eastern Finland, P. O. Box $111 \mathrm{Fl}-80101$ Joensuu, Finland

Full list of author information is available at the end of the article
The SPPs, customarily treated as monochromatic (fully coherent), greatly modify the statistical properties of optical near fields [12-16]. However, there is an emerging recognition that partial coherence can be an important degree of freedom in controlling SPP distributions $[17,18]$. A crucial step was taken quite recently by introducing a general theoretical framework to customize the coherence features of polychromatic SPPs in the Kretschmann setup [19] and a protocol based on point-dipole scattering to recover statistically stationary SPP correlations from far-field spectral information was put forward [20]. Further, a general coherent pseudomode representation of partially coherent SPP fields was advanced [21] and planar axicon-like and lattice-type SPP fields of varying coherence states were analyzed [22, 23]. Such plasmon coherence engineering is instrumental for synthesizing structured SPP fields with desired spatiotemporal coherence and polarization properties.

In this work we investigate numerically the scattering of SPPs by a metallic nanoparticle for three slightly different wavelengths of light. The analysis is carried out in two spatial dimensions and the nanoparticle has a square shape, so we call it a nanostripe (the system may be 
viewed as uniform perpendicular to the plane of analysis). The SPPs are excited onto a metal-air interface in a Kretschmann geometry and subsequently interact with the metallic nanostripe a short distance away. The nanostripe has a dual physical effect: firstly, back-reflection creates standing spectral wave patterns between the SPP origin and the nanostripe, and secondly, part of the SPP energy is scattered into the far zone of the nanoscatterer. A square nanostripe is relatively easy to handle numerically. Moreover, it must be appropriately small to yield forward scattering (yet large enough for sufficient scattering efficiency). From the far-field spectrum we may deduce the relative strengths of the spectral SPP components, which in turn determine the spatiotemporal coherence properties of the polychromatic standing SPP pattern. It is demonstrated that the nanostripe reflections profoundly alter the spatial and temporal coherence, even though the SPP field is fully coherent at each frequency.

\section{Methods}

A schematic illustration of the system under study is shown in Fig. 1. Stationary, polychromatic light incident from a dielectric medium in a Kretschmann configuration creates a surface plasmon polariton field $\mathbf{E}_{\mathrm{p}}$ that propagates along the metal-air interface towards a nanostripe N. Most of $\mathbf{E}_{\mathrm{p}}$ is reflected at $\mathrm{N}$ as a backward-propagating plasmon field $\mathbf{E}_{\mathrm{r}}$, while a small part is scattered into the half-space above the metal in the form of an effectively freely propagating electric field $\mathbf{E}_{\mathrm{s}}$. A detector $\mathrm{D}$ in the far zone of the nanostripe $\mathrm{N}$ measures the spectrum of the scattered light, from which the spectral amplitudes of $\mathbf{E}_{\mathrm{p}}$ and thereby all the spatiotemporal coherence (and polarization) properties of the superposition surface plasmon field can be inferred.

\section{Theory}

\section{Surface plasmon polaritons}

In the absence of the nanostripe the metal film on a dielectric medium is homogeneous and characterized by a complex relative permittivity $\epsilon_{\mathrm{r}}(\omega)$, accounting for dispersion and absorption. A p-polarized (TM), statistically stationary, tailored illumination excites a polychromatic SPP field on the metal-air interface (see Fig. 1). The film thickness $h$ is large enough so that coupling among the plasmon modes at the two metal surfaces can be neglected. The spatial electric part of the SPP field in air, at point $\mathbf{r}=(x, z)$, frequency $\omega$, and propagating in the positive $x$ direction, may then be expressed as $[19,20]$

$$
\mathbf{E}_{\mathbf{p}}(\mathbf{r}, \omega)=E(\omega) \hat{\mathbf{p}}(\omega) \mathrm{e}^{\mathrm{i} \mathbf{k}(\omega) \cdot \mathbf{r}},
$$

where $E(\omega)$ is the complex field amplitude at the origin $(\mathbf{r}=0)$, and

$$
\begin{aligned}
\mathbf{k}(\omega) & =k_{x}(\omega) \hat{\mathbf{e}}_{x}+k_{z}(\omega) \hat{\mathbf{e}}_{z} \\
\hat{\mathbf{p}}(\omega) & =\left[\mathbf{k}_{\mathrm{p}}(\omega) \times \hat{\mathbf{e}}_{y}\right] /\left|\mathbf{k}_{\mathrm{p}}(\omega)\right|=p_{x}(\omega) \hat{\mathbf{e}}_{x}+p_{z}(\omega) \hat{\mathbf{e}}_{z}
\end{aligned}
$$

are the wave vector and the unit-normalized polarization vector, respectively, and $\hat{\mathbf{e}}_{x}, \hat{\mathbf{e}}_{y}$, and $\hat{\mathbf{e}}_{z}$ are the Cartesian unit vectors. The wave-vector components in Eq. (2) read as $[1,2]$

$$
\begin{aligned}
& k_{x}(\omega)=\frac{\omega}{c} \sqrt{\frac{\epsilon_{\mathrm{r}}(\omega)}{\epsilon_{\mathrm{r}}(\omega)+1}}, \\
& k_{z}(\omega)=\frac{\omega}{c} \sqrt{\frac{1}{\epsilon_{\mathrm{r}}(\omega)+1}},
\end{aligned}
$$

where $c$ is the speed of light in free space.

When the nanostripe $\mathrm{N}$ is present, it serves as a barrier from which the SPP reflects back and may, to a good accuracy, be represented as

$$
\mathbf{E}_{\mathbf{r}}(\mathbf{r}, \omega)=E_{\mathrm{r}}(\omega) \hat{\mathbf{p}}_{\mathrm{r}}(\omega) \mathrm{e}^{\mathrm{i} \mathbf{k}_{\mathbf{r}}(\omega) \cdot\left(\mathbf{r}-d \hat{\mathbf{e}}_{x}\right)},
$$

where $E_{\mathrm{r}}(\omega)$ is the complex amplitude of the reflected SPP at the nanostripe $[\mathbf{r}=(d, 0)]$. Further, from the properties of SPPs it follows that $[22,23]$

$$
\begin{aligned}
& \mathbf{k}_{\mathrm{r}}(\omega)=-k_{x}(\omega) \hat{\mathbf{e}}_{x}+k_{z}(\omega) \hat{\mathbf{e}}_{z}, \\
& \hat{\mathbf{p}}_{\mathrm{r}}(\omega)=-p_{x}(\omega) \hat{\mathbf{e}}_{x}+p_{z}(\omega) \hat{\mathbf{e}}_{z} .
\end{aligned}
$$

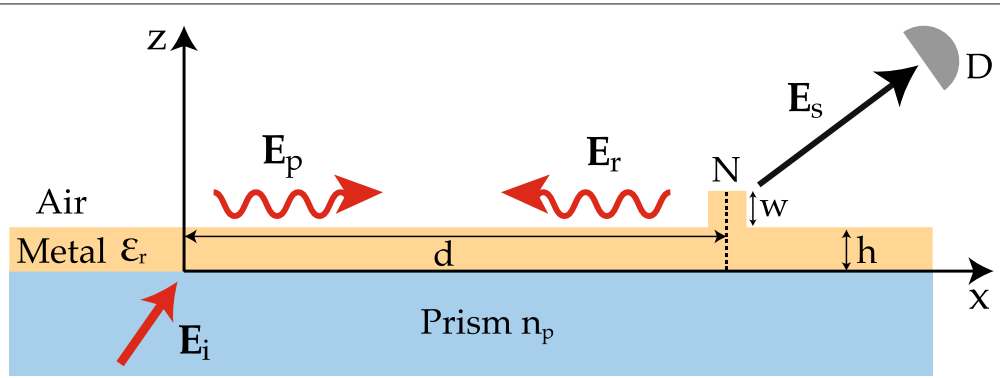

Fig. 1 (Color online) Schematic diagram of SPP generation and scattering by a nanostripe in the Kretschmann configuration. The field $\mathbf{E}_{\mathbf{i}}$ is an excitation wave, $\mathbf{E}_{\mathrm{p}}$ and $\mathbf{E}_{\mathrm{r}}$ are forward- and backward-going SPPs on the metal-air interface, $\mathbf{E}_{\mathrm{s}}$ is a field scattered by the nanostripe $\mathrm{N}$ into free space, and D is a detector. The coordinate axes and the symbols specifying the system geometry and material properties are indicated in the figure 
Considering the SPP propagation at $z=0$, we may estimate

$$
\begin{aligned}
E_{\mathrm{r}}(\omega) & =r(\omega) E(\omega) \mathrm{e}^{\mathrm{i} k_{x}(\omega) d}, \\
r(\omega) & =\frac{1-n(\omega)}{1+n(\omega)},
\end{aligned}
$$

where $r(\omega)$ is the field reflection coefficient. In it $n(\omega)=$ $\sqrt{\epsilon_{\mathrm{r}}(\omega)}$ is the (complex) refractive index of the metal nanostripe [24]. The coefficient $r(\omega)$ corresponds to reflection of a normally incident plane wave, as is typically the case with SPPs [22].

From Eqs. (1) and (6) we now readily find for the total SPP field between the excitation point and the nanostripe $\mathrm{N}$ the expression

$$
\begin{aligned}
\mathbf{E}_{\mathrm{SPP}}(\mathbf{r}, \omega)= & E(\omega) \mathrm{e}^{\mathrm{i} k_{z}(\omega) z}\left[\hat{\mathbf{p}}(\omega) \mathrm{e}^{\mathrm{i} k_{x}(\omega) x}\right. \\
& \left.+r(\omega) \mathrm{e}^{\mathrm{i} 2 k_{x}(\omega) d} \hat{\mathbf{p}}_{\mathbf{r}}(\omega) \mathrm{e}^{-\mathrm{i} k_{x}(\omega) x}\right],
\end{aligned}
$$

or explicitly in the component form

$$
E_{\mathrm{SPP} x}(\mathbf{r}, \omega)=E(\omega) \mathrm{e}^{\mathrm{i} k_{z}(\omega) z} p_{x}(\omega)\left[\mathrm{e}^{\mathrm{i} k_{x}(\omega) x}-r(\omega) \mathrm{e}^{\mathrm{i} 2 k_{x}(\omega) d} \mathrm{e}^{-\mathrm{i} k_{x}(\omega) x}\right],
$$

$E_{\mathrm{SPP} z}(\mathbf{r}, \omega)=E(\omega) \mathrm{e}^{\mathrm{i} k_{z}(\omega) z} p_{z}(\omega)\left[\mathrm{e}^{\mathrm{i} k_{x}(\omega) x}+r(\omega) \mathrm{e}^{\mathrm{i} 2 k_{x}(\omega) d} \mathrm{e}^{-\mathrm{i} k_{x}(\omega) x}\right]$.

The relative sizes of these components depend on the metal and the frequency, which determine the SPP wave vector $\mathbf{k}(\omega)$ and polarization vector $\hat{\mathbf{p}}(\omega)$.

\section{Spatiotemporal coherence}

We view the SPP field in Eq. (11) as a realization of a statistically stationary ensemble and compute the crossspectral density matrix as $[19,20]$

$$
\mathbf{W}\left(\mathbf{r}_{1}, \mathbf{r}_{2}, \omega\right)=\left\langle\mathbf{E}_{\mathrm{SPP}}^{*}\left(\mathbf{r}_{1}, \omega\right) \mathbf{E}_{\mathrm{SPP}}^{\mathrm{T}}\left(\mathbf{r}_{2}, \omega\right)\right\rangle,
$$

where the asterisk and superscript $\mathrm{T}$ denote complex conjugation and matrix transpose, respectively, and the angle brackets stand for ensemble averaging. Since $E(\omega)$ is the only random quantity, the polychromatic SPP field clearly is fully coherent in the space-frequency domain. Its spatiotemporal coherence is obtained from the generalized Wiener-Khintchine theorem [25]

$$
\boldsymbol{\Gamma}\left(\mathbf{r}_{1}, \mathbf{r}_{2}, \tau\right)=\int_{0}^{\infty} \mathbf{W}\left(\mathbf{r}_{1}, \mathbf{r}_{2}, \omega\right) \mathrm{e}^{-\mathrm{i} \omega \tau} \mathrm{d} \omega
$$

in which $\boldsymbol{\Gamma}\left(\mathbf{r}_{1}, \mathbf{r}_{2}, \tau\right)$ is the mutual coherence matrix and $\tau$ is a time difference. Expression (15), with $\mathbf{E}_{\mathrm{SPP}}(\mathbf{r}, \omega)$ given by Eq. (11), is valid everywhere between the SPP creation point and the nanostripe $\mathrm{N}$, for statistically stationary excitations of any spectral distribution.

\section{Scattered far field}

Interaction of $\mathbf{E}_{\mathrm{p}}$ with the nanostripe $\mathrm{N}$ produces, besides the reflected SPP $\mathbf{E}_{\mathrm{r}}$ of Eq. (6), also a field scattered into the half-space $z>0$. If the nanostripe is sufficiently small, it behaves much like a point scatterer [26, 27] (albeit in a 2D space). In the far-zone the scattered electric field is normal to the propagation direction and has spherical wave fronts. Within a good approximation, we may thus write for the scattered far-field amplitude the expression [28]

$$
E_{\mathrm{S}}(s, \phi, \omega)=r(\phi, \omega) E(d, \omega) \frac{\mathrm{e}^{\mathrm{i}(\omega / c) s}}{\sqrt{s}},
$$

where $r(\phi, \omega)$ is the scattering coefficient with $\phi$ representing the angle between the $x$ axis and the scattering direction. Further, $E(d, \omega)$ is the amplitude of the forwardgoing SPP at the location of the nanostripe $\mathrm{N}$ and $s$ is the distance between $\mathrm{N}$ and the observation point. Denoting the spectral intensities at detector $\mathrm{D}$ and nanostripe $\mathrm{N}$ by $I_{\mathrm{s}}(\phi, \omega)=\left\langle\left|E_{\mathrm{s}}(s, \phi, \omega)\right|^{2}\right\rangle$ and $I(d, \omega)=\left\langle|E(d, \omega)|^{2}\right\rangle$, respectively, it follows from Eq. (16) that

$$
s I_{\mathrm{s}}(\phi, \omega)=|r(\phi, \omega)|^{2} I(d, \omega),
$$

implying that the scattered far-zone intensity multiplied by distance $s$ is a constant that, in general, depends on the scattering direction and the frequency.

\section{Simulation}

The simulations of vectorial SPP fields on the metallic surface and their scattering from the nanostripe are performed in $2 \mathrm{D}$ by utilizing in-house numerical codes based on the Fourier modal method [29], as well as COMSOL Multiphysics software that employs the finite element method. The SPP excitation in the Kretschmann geometry takes place by means of a perfectly phase-matched focused beam at each frequency. In all our simulations the two different computational methods lead to substantially similar results.

We illustrate in Fig. 2 the salient features of the simulations with a monochromatic excitation. As in all subsequent analyses, the metal layer thickness $h=65 \mathrm{~nm}$ and the nanostripe side length $w=80 \mathrm{~nm}$. The material in both the layer and the nanostripe is gold $(\mathrm{Au})$, while the dielectric prism consists of glass $\left(\mathrm{SiO}_{2}\right)$. Figure 2a demonstrates clearly the existence of a standing SPP wave pattern left of the nanostripe $\mathrm{N}$ (since the plasmon survival length is large compared to the separation $d$ ), and Fig. 2b shows a series of cone-like radiation lobes (due to the square shape of the scatterer) emerging from $\mathrm{N}$ with their strength decreasing as the scattering angle increases. We note that this latter figure is plotted starting from slightly above the metal surface so that the standing waves are not, for the most part, visible in it anymore. 

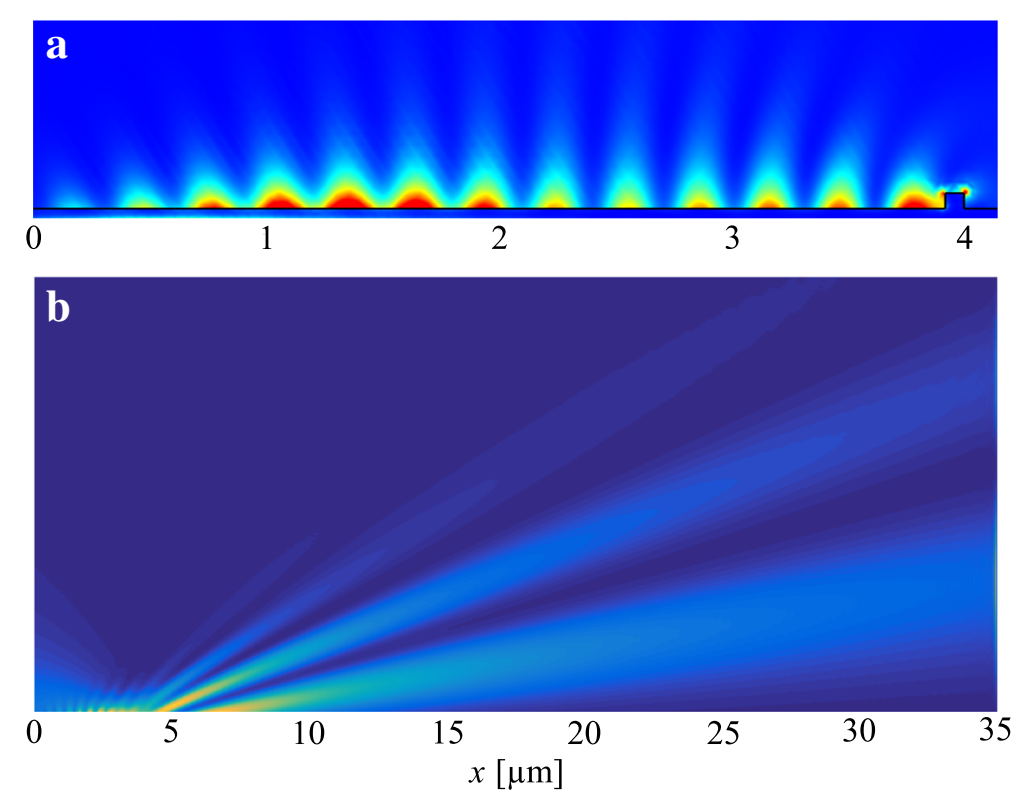

Fig. 2 (Color online) Simulation of SPP creation and nanoscattering, with a a standing wave pattern between the excitation point and the nanostripe, and $\mathbf{b}$ radiation into the far zone in air beyond the nanostripe. The nanostripe is located at the distance of $4 \mu \mathrm{m}$ from the SPP excitation point. The (free-space) wavelength is $\lambda=633 \mathrm{~nm}$. The spatial scales and color schemes in $\mathbf{a}$ and $\mathbf{b}$ are different to demonstrate the weak far-field scattering

\section{Results and discussion}

\section{Spectral intensity determination}

Following a plasmon coherence engineering approach [19] in the Kretschmann setup we generate the SPPs at three (free-space) wavelengths $\lambda_{j}, j=1,2,3$, in the red region of the spectrum (see Table 1). The excitation angles in the prism (relative to metal-slab normal, see Fig. 1) are obtained from $n_{\mathrm{p}}(\omega) \sin \theta(\omega)=$ $\operatorname{Re}\left\{\left[\epsilon_{\mathrm{r}}(\omega) /\left(\epsilon_{\mathrm{r}}(\omega)+1\right)\right]^{1 / 2}\right\}$, where $n_{\mathrm{p}}(\omega)$ is the refractive index of $\mathrm{SiO}_{2}$ and $\epsilon_{\mathrm{r}}(\omega)$ is the dielectric permittivity of $\mathrm{Au}$. The angles, together with the SPP wavelengths $\lambda_{\mathrm{SPP}}(\omega)=2 \pi / k_{x}^{\prime}(\omega)$ and propagation lengths $l_{\mathrm{SPP}}(\omega)=$ $1 / 2 k_{x}^{\prime \prime}(\omega)$, with the prime and double prime denoting the real and imaginary part, respectively, as well as the SPP life times $t_{\mathrm{SPP}}(\omega)=l_{\mathrm{SPP}}(\omega) / v_{\mathrm{g}}(\omega)$, where $v_{\mathrm{g}}(\omega)$ is the group velocity, at the gold-air interface are listed in Table 1 . The material parameters here, and henceforth, are based on [30].

In Fig. 3 we display the intensity profiles of the three spectral SPP components along the metal surface in the absence of the nanostripe, obtained by simulations when

Table 1 SPP excitation and characteristics at three wavelengths on an air-Au interface

\begin{tabular}{llllll}
\hline$j$ & $\lambda[\mathrm{nm}]$ & $\theta\left[^{\circ}\right]$ & $\lambda_{\text {SPP }}[\mathrm{nm}]$ & ISPP $[\mu \mathrm{m}]$ & $t_{\text {SPP }}[\mathrm{ps}]$ \\
\hline 1 & 633 & 45.83 & 603.1 & 12.9 & 0.045 \\
2 & 642 & 45.70 & 613.4 & 14.9 & 0.052 \\
3 & 650 & 45.60 & 622.3 & 17.0 & 0.059 \\
\hline
\end{tabular}

the excitation field strengths are equal. From these plots we deduce, firstly, the (maximum) spectral SPP intensities $I(\omega)=\left\langle|E(\omega)|^{2}\right\rangle$ at excitation and, secondly, the spectral intensity decay factors $\exp \left[-l_{\mathrm{SPP}}(\omega) d\right]$ due to SPP propagation to the nanostripe $\mathrm{N}$ [see Eq. (9)]. The intensity ratios $I\left(\lambda_{j}\right) / I\left(\lambda_{1}\right), j=1,2,3$, of the respective spectral SPP constituents obtained by such direct simulation are given in Table 2.

According to Eq. (17), the scattered far-field intensity multiplied by the distance $s$ from the nanostripe $\mathrm{N}$ acquires a constant value, independent of $s$. This provides, in principle, an experimental means of recovering the spectral SPP intensities at the excitation point. With reference to Fig. 2, we evaluate the scattered field intensities along a straight line from $\mathrm{N}$ into the far zone, in the center of the second scattering cone. Multiplied by $s$, this gives the left-hand side of Eq. (17) for the three wavelengths, and their behavior is shown in the inset to Fig. 3. Taking the scattering coefficients $|r(\phi, \omega)|^{2}$ equal for all wavelengths, we thereby obtain the spectral SPP intensity

Table 2 Normalized spectral intensities at SPP excitation in the Kretschmann geometry, obtained by direct simulation and from far-field scattering by a nanostripe

\begin{tabular}{lll}
\hline Wavelength $[\mathrm{nm}]$ & Direct simulation & Far-field scattering \\
\hline 633 & 1.0 & 1.0 \\
642 & 0.880 & 0.879 \\
650 & 0.790 & 0.781 \\
\hline
\end{tabular}




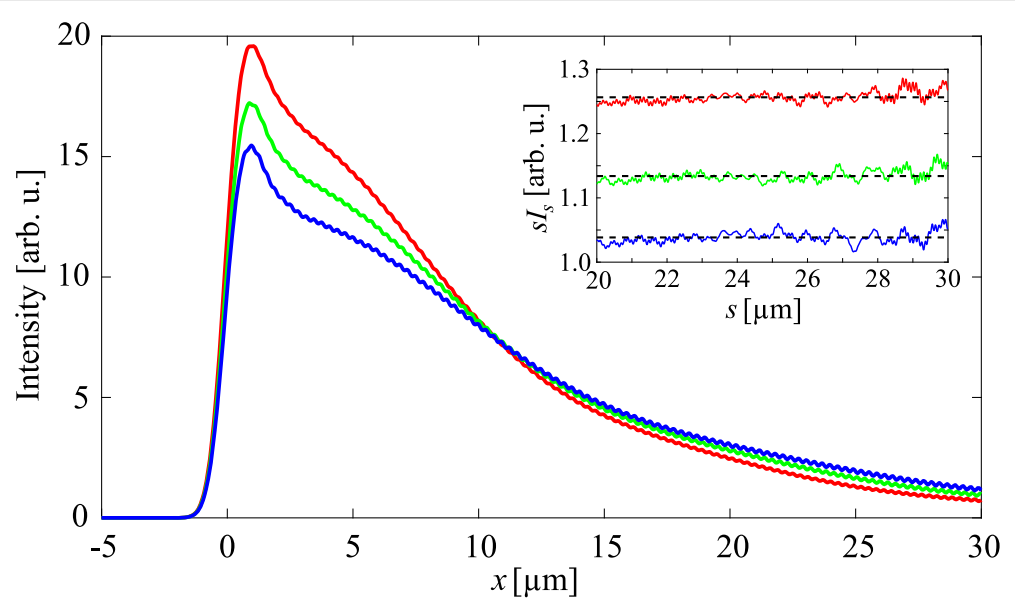

Fig. 3 (Color online) SPP intensities at $z=0$ for $\lambda=633 \mathrm{~nm}$ (red), $\lambda=642 \mathrm{~nm}$ (green), and $\lambda=650 \mathrm{~nm}$ (blue). The inset shows the far-field scattered intensities times the scattering distance $s$, as a function of $s$ [see Eq. (17)]

ratios at the nanostripe $\mathrm{N}$. On further accounting for the different SPP propagation losses we again recover the relative intensities $I\left(\lambda_{j}\right) / I\left(\lambda_{1}\right), j=1,2,3$, at the excitation point. These values, obtained via far-field scattering, are likewise given in Table 2. We observe that the agreement between the results from direct simulations and from farfield scattering is excellent, thus confirming the validity of the approach.

\section{Spatial coherence}

Since we are considering SPPs on a gold-air interface at red wavelengths, the transverse electric field component is by far dominant [i.e., $\left.\left|p_{z}(\omega)\right| \gg\left|p_{x}(\omega)\right|\right]$ and we may restrict spatial coherence analyses to Eq. (13). If shorter wavelengths or other media are involved, the complete vectorial SPP expression in Eq. (11) is required [22] and, consequently, the concept of electromagnetic degree of coherence would have to be employed [25]. As the SPPs are strongly confined onto the metal surface, it is reasonable to take $z=0$ throughout the coherence assessment. Substitution of Eq. (13) into Eq. (14) then yields

$$
\begin{aligned}
W\left(x_{1}, x_{2}, \omega\right) & =I(\omega)\left\{\mathrm{e}^{-\mathrm{i}\left[k_{x}^{*}(\omega) x_{1}-k_{x}(\omega) x_{2}\right]}+a(\omega) \mathrm{e}^{-\mathrm{i}\left[k_{x}^{*}(\omega) x_{1}+k_{x}(\omega) x_{2}\right]}\right. \\
& \left.+a^{*}(\omega) \mathrm{e}^{\mathrm{i}\left[k_{x}^{*}(\omega) x_{1}+k_{x}(\omega) x_{2}\right]}+|a(\omega)|^{2} \mathrm{e}^{\mathrm{i}\left[k_{x}^{*}(\omega) x_{1}-k_{x}(\omega) x_{2}\right]}\right\},
\end{aligned}
$$

where we have set $p_{z}(\omega)=1, I(\omega)=\left\langle|E(\omega)|^{2}\right\rangle$, and $a(\omega)=$ $r(\omega) \exp \left[\mathrm{i} 2 k_{x}(\omega) d\right]$. Since the SPP excitation takes place at three frequencies $\omega_{j}, j=1,2,3$, the mutual coherence function from Eq. (15) now becomes

$$
\Gamma\left(x_{1}, x_{2}, \tau\right)=\sum_{j=1}^{3} W\left(x_{1}, x_{2}, \omega_{j}\right) \mathrm{e}^{-\mathrm{i} \omega_{j} \tau} .
$$

The complex degree of space-time coherence then is defined as

$$
\gamma\left(x_{1}, x_{2}, \tau\right)=\frac{\Gamma\left(x_{1}, x_{2}, \tau\right)}{\left[\Gamma\left(x_{1}, x_{1}, 0\right) \Gamma\left(x_{2}, x_{2}, 0\right)\right]^{1 / 2}},
$$

and it satisfies $0 \leq\left|\gamma\left(x_{1}, x_{2}, \tau\right)\right| \leq 1$. We obtain from Eqs. (18) and (19)

$$
\begin{gathered}
\Gamma(x, x, 0)=\sum_{j=1}^{3} I\left(\omega_{j}\right) G\left(x, \omega_{j}\right), \\
G(x, \omega)=\mathrm{e}^{-2 k_{x}^{\prime \prime}(\omega) x}+2 \operatorname{Re}\left[a(\omega) \mathrm{e}^{-i 2 k_{x}^{\prime}(\omega) x}\right]+|a(\omega)|^{2} \mathrm{e}^{2 k_{x}^{\prime \prime}(\omega) x},
\end{gathered}
$$

for the total SPP intensity at point $\mathbf{r}=(x, 0)$. Using Eq. (20), we consider first the spatial coherence function $\gamma\left(x_{1}, x_{2}, 0\right)$ on the metal surface.

If the distance from SPP creation to the nanostripe N is small compared to the plasmon survival lengths, the principal features of the spatial coherence do not effectively depend on the point about which the coherence is evaluated. Hence, in Fig. 4a we display the spatial coherence as a function of $x_{2}$, with $x_{1}=0, d=4 \mu \mathrm{m}$, and the spectral intensity ratios taken from Table 2 . The degree of spatial coherence is seen to have certain characteristic properties and it differs considerably from the spatial coherence in the absence of the nanostripe, when it remains generally high (dashed curve). We observe, firstly, that the spatial coherence has a (nearly) periodic structure, with the period determined by the SPP wave vectors. The periodic behavior of the degree of coherence has similarities with the intensity fluctuation of the standing wave [Fig. 2a]. The differences arise from the interplay of multiple frequencies, complex permittivities, and propagation distances of the coherence function. Secondly, the maxima reach very close to unity, while the minima are as low as only about 0.3 . We emphasize that since the SPP field is stationary (frequency components are uncorrelated), the 

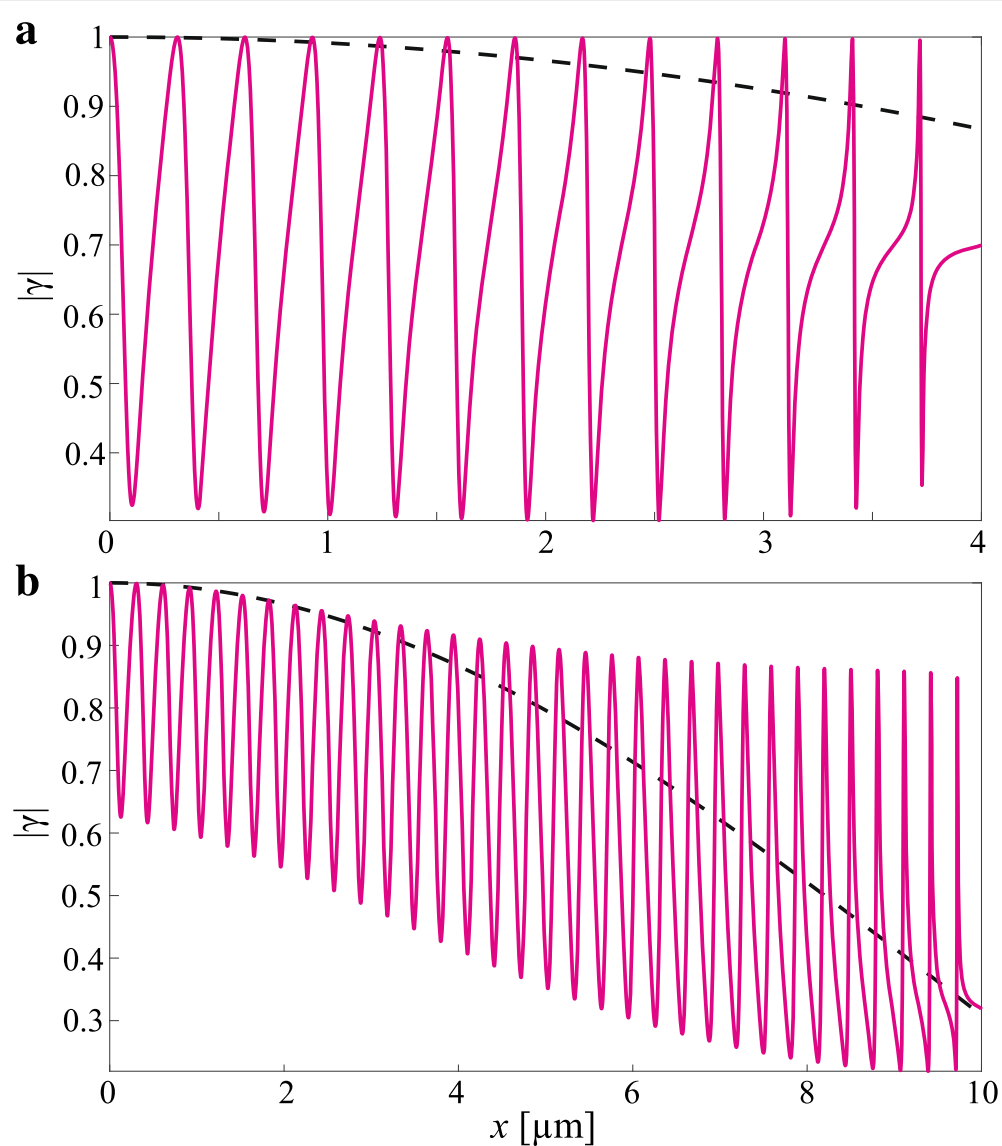

Fig. 4 (Color online) Degree of spatial coherence $|\gamma(0, x, 0)|$ associated with the total SPP field on the metal surface with $\mathbf{a} d=4 \mu \mathrm{m}$, and $\mathbf{b}$ $d=10 \mu \mathrm{m}$. The spectral intensity ratios are taken from Table 2 . The dashed curve corresponds to the degree of spatial coherence in the absence of the nanostripe

variation of the degree of spatial coherence is not a consequence of wave beating but follows from the statistical similarity [31] of the total SPP fields at the various points. When the nanostripe is further away, at $d=10 \mu \mathrm{m}$, the periodicity of the degree of spatial coherence remains but the maxima and minima vary more due to the different decay factors of the spectral constituents, as is illustrated in Fig. 4b.

\section{Temporal coherence}

For the temporal coherence of the polychromatic SPP field, at point $\mathbf{r}=(x, 0)$ on the metal surface, we find from Eq. (20)

$$
\gamma(x, x, \tau)=\frac{\Gamma(x, x, \tau)}{\Gamma(x, x, 0)}
$$

where $\Gamma(x, x, 0)$ is given by Eq. (21) and the numerator takes on the form

$$
\Gamma(x, x, \tau)=\sum_{j=1}^{3} I\left(\omega_{j}\right) G\left(x, \omega_{j}\right) \mathrm{e}^{-\mathrm{i} \omega_{j} \tau} .
$$

where $G\left(x, \omega_{j}\right)$ is obtained from Eq. (22).

As with spatial coherence, the typical features of the temporal coherence depend only weakly (due to the SPPs) on the position at the metal surface. We illustrate in Figs. $5 \mathrm{a}$ and $5 \mathrm{~b}$ the degree of temporal coherence at the SPP excitation point, when the nanostripe $\mathrm{N}$ is located at $d=4 \mu \mathrm{m}$ and $d=10 \mu \mathrm{m}$, respectively, and the spectral intensities correspond to the values in Table 2. The near periodicity of the coherence degree, originating from the three spectral SPP wave vectors, is again clearly visible in both figures. When the separation of the nanostripe from the SPP excitation point is short compared to the SPP propagation lengths, the degree of temporal coherence remains generally high at all times [see Fig. 5a], in contrast to the situation in the absence of the nanoscatterer when the coherence degree varies considerably in time (dashed curve). The contributions due to reflection increase the temporal coherence on average, but the details again specifically depend on the frequencies, complex permittivities, and the nanoscatterer position. If the distance to the nanostripe is increased, the influence of 

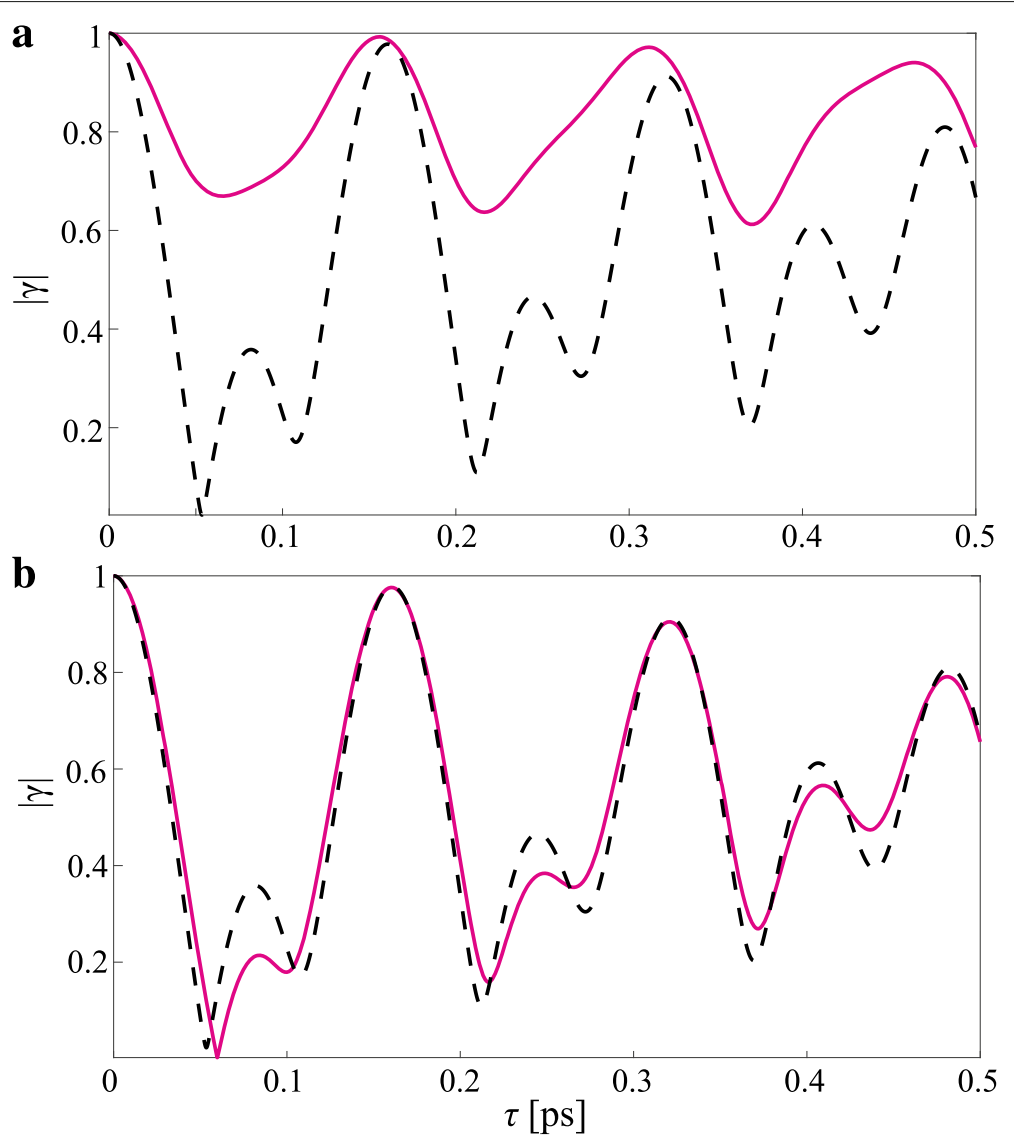

Fig. 5 (Color online) Degree of temporal coherence $|\gamma(0,0, \tau)|$ of the total SPP field, when $\mathbf{a} d=4 \mu \mathrm{m}$ and $\mathbf{b} d=10 \mu \mathrm{m}$, and the relative spectral intensities are those in Table 2. The dashed curve shows the degree of temporal coherence in the absence of the nanostripe

the reflected SPPs in the interference pattern diminishes and the degrees of temporal coherence with and without the nanostripe approach each other [see Fig. 5b]. If there are no reflected SPPs, the degree of temporal coherence at the excitation point is solely determined by the Fourier transforms of the spectral intensities. Comparison of Figs. 5a and 5b with the SPP life times in Table 1 indicates that owing to the continual (stationary) excitation of the SPPs, the temporal coherence persists way beyond the sub-ps life times and coherence variations in time arise from the lack of correlations between the different frequencies.

\section{Conclusions}

In summary, we have studied by numerical simulations the scattering of SPPs from a metallic nanostripe. The SPPs constitute a polychromatic, statistically stationary, electromagnetic surface field, excited onto an air-metal interface in the Kretschmann configuration through exact phase matching at each frequency. For the simulation we use both in-house codes and commercial software.
By comparison with direct calculations we demonstrate, first of all, that detection of the spectrum in the farzone of the nanostripe allows one to recover the intensities of the spectral SPP constituents and thereby deduce the complete spatiotemporal coherence properties of the SPP field. Secondly, our analyses and simulations show that the presence of the nanostripe leads to the creation of a standing SPP pattern whose spatial and temporal coherence properties differ significantly from those in the absence of the nanoscatterer. Characteristic features of coherence include near periodicity originating from statistical similarity and variations of maxima and minima due to spectrally dependent SPP survival lengths. And finally, although we explicitly assessed red spectral components in gold only, the method is general, i.e., the materials and excitation spectra can be arbitrary providing an opportunity to judiciously tailor the SPP standing-field coherence properties. Such plasmon coherence engineering may find uses in controlled excitation of particles and clusters and in emerging applications of nanoplasmonics technology. 
Acknowledgements

Not applicable.

\section{Funding}

This work was supported by the Natural Science and Engineering Research Council (NSERC) of Canada (Grant No. RGPIN-2018-05497), the Academy of Finland (Project No. 310511), and the Joensuu University Foundation.

\section{Availability of supporting data}

Not applicable.

\section{Authors' contributions}

The original ideas and results emerged from discussions among all the authors. SD and KS performed the calculations and assisted SAP and ATF in writing the manuscript.

\section{Authors' information}

Not applicable.

\section{Competing interests}

The authors declare that they have no competing interests.

\section{Publisher's Note}

Springer Nature remains neutral with regard to jurisdictional claims in published maps and institutional affiliations.

\section{Author details}

${ }^{1}$ Institute of Photonics, University of Eastern Finland, P. O. Box 111 Fl-80101 Joensuu, Finland. ${ }^{2}$ Department of Electrical and Computer Engineering, Dalhousie University, Halifax, Nova Scotia B3J 2X4, Canada. ${ }^{3}$ Department of Physics and Atmospheric Science, Dalhousie University, Halifax, Nova Scotia B3H 2R2, Canada.

Received: 6 November 2018 Accepted: 19 March 2019

Published online: 04 April 2019

\section{References}

1. Maier, SA: Plasmonics: Fundamentals and Applications. Springer, Berlin (2007)

2. Novotny, L, Hecht, B: Principles of Nano-Optics. 2nd edition. Cambridge University Press, Cambridge (2012)

3. Fang, Y, Sun, M: Nanoplasmonic waveguides: towards applications in integrated nanophotonic circuits. Light Sci. Appl. 4, e294 (2015)

4. Anker, JN, Hall, WP, Lyandres, O, Shah, NC, Zhao, J, Van Duyne, RP: Biosensing with plasmonic nanosensors. Nat. Mater. 7, 442-453 (2008)

5. O'Connor, D, Zayats, AV: The third plasmonic revolution. Nat. Nanotech. $\mathbf{5}$ 482-483 (2010)

6. Guo, $X, M a, Y$, Wang, $Y$, Tong, L: Nanowire plasmonic waveguides, circuits and devices. Laser Photon. Rev. 7, 855-881 (2013)

7. Faryad, M, Lakhtakia, A: Grating-coupled excitation of multiple surface plasmon-polariton waves. Phys. Rev. A. 84, 033852 (2011)

8. Raether, H: Surface Plasmons on Smooth and Rough Surfaces and on Gratings. Springer, Berlin (1988)

9. Evlyukhin, AB, Brucoli, G, Martín-Moreno, L, Bozhevolnyi, SI, García-Vidal, FJ: Surface plasmon polariton scattering by finite-size nanoparticles. Phys. Rev. B. 76, 075426 (2007)

10. Sánchez-Gil, JA, Maradudin, AA: Surface-plasmon polariton scattering from a finite array of nanogrooves/ridges: Efficient mirrors. Appl. Phys. Lett. 86, 251106 (2005)

11. Leppänen, L-P, Saastamoinen, K, Lehtolahti, J, Friberg, AT, Setälä, T: Detection of partial polarization of light beams with dipolar nanocubes. Opt. Express. 24, 1472-1479 (2016)

12. Shchegrov, AV, Joulain, $K$, Carminati, $R$, Greffet, J-J: Near-field spectral effects due to electromagnetic surface excitations. Phys. Rev. Lett. 85, 1548-1551 (2000)

13. Setälä, T, Kaivola, M, Friberg, AT: Degree of polarization in near fields of thermal sources: effects of surface waves. Phys. Rev. Lett. 88, 123902 (2002)

14. Gan, CH, Gbur, G, Visser TD: Surface plasmons modulate the spatial coherence of light in Young's interference experiment. Phys. Rev. Lett. 98 $043908(2007)$
15. Divitt, S, Frimmer, M, Visser, TD, Novotny, L: Modulation of optical spatial coherence by surface plasmon polaritions. Opt. Lett. 41, 3094-3097 (2016)

16. Morrill, D, Li, D, Pacifici, D: Measuring subwavelength spatial coherence with plasmonic interferometry. Nat. Photon. 10, 681-687 (2016)

17. Laverdant, J, Aberra Guebrou, S, Bessueille, F, Symonds, C, Bellessa, J: Leakage interferences applied to surface plasmon analysis. J. Opt. Soc Am. A. 31, 1067-1073 (2014)

18. Norrman, A, Setälä, T, Friberg, AT: Partial coherence and polarization of a two-mode surface-plasmon polariton field at a metallic nanoslab. Opt. Express. 23, 20696-20714 (2015)

19. Norrman, A, Ponomarenko, SA, Friberg, AT: Partially coherent surface plasmon polaritons. EPL. 116, 64001 (2016)

20. Chen, Y, Norrman, A, Ponomarenko, SA, Friberg, AT: Plasmon coherence determination by nanoscattering. Opt. Lett. 42, 3279-3282 (2017)

21. Mao, H, Chen, Y, Ponomarenko, SA, Friberg, AT: Coherent pseudo-mode representation of partially coherent surface plasmon polaritons. Opt. Lett. 43, 1395-1398 (2018)

22. Chen, Y, Norrman, A, Ponomarenko, SA, Friberg, AT: Partially coherent axiconic surface plasmon polariton fields. Phys. Rev. A. 97, 041801 (R) (2018)

23. Chen, Y, Norrman, A, Ponomarenko, SA, Friberg, AT: Coherence lattices in surface plasmon polariton fields. Opt. Lett. 43, 3429-3432 (2018)

24. Hecht, E: Optics. 4th edition. Addison-Wesley, San Francisco (2002)

25. Friberg, AT, Setälä, T: Electromagnetic theory of optical coherence (invited). J. Opt. Soc. Am. A. 33, 2431-2442 (2016)

26. Bohren, CF, Huffman, DR: Absorption and Scattering of Light by Small Particles. Wiley, New York (1998)

27. Massa, E, Maier, SA, Giannini, V: An analytical approach to light scattering from small cubic and rectangular cuboidal nanoantennas. New J. Phys. 15, 063013 (2013)

28. Friberg, AT, Reng, N: Wide-angle reciprocity relations between near and far fields. Proc SPIE. 2870, 154-162 (1996)

29. Turunen, J: Diffraction theory of microrelief gratings. In: Herzig, HP, editor: Micro-optics: Elements, Systems and Applications. Taylor and Francis, London, (1997)

30. Weast, RC (Ed): CRC Handbook of Chemistry and Physics. CRC Press, Boca Raton (1984)

31. Ponomarenko SA, Roychowdhury, H, Wolf, E: Physical significance of complete spatial coherence of optical fields. Phys. Lett. A. 345, 10-12 (2005)

\section{Submit your manuscript to a SpringerOpen ${ }^{\circ}$ journal and benefit from:}

- Convenient online submission

- Rigorous peer review

Open access: articles freely available online

- High visibility within the field

- Retaining the copyright to your article

Submit your next manuscript at $\gg$ springeropen.com 